\title{
Investigation On Psychological Status of Patients With Cervical Precancerous Lesions And Cancer Among Han And Ethnic Minority In Yunnan Province of China
}

\author{
Min Zhao \\ Yunnan Cancer Hospital \\ Dan Liu \\ Chongqing Medical University \\ Rong-Yan Gu \\ Yunnan Cancer Hospital \\ Hong-Tao Lei \\ Kunming Medical University \\ Shao Zhang \\ Yunnan Cancer Hospital
}

Song-Rui Ding

Yunnan Cancer Hospital

Lei Luo

Yunnan Cancer Hospital

Meng-Jiao Zhang

Yunnan Cancer Hospital

Guo-Yu Ma

Yunnan Cancer Hospital

Zheng Li ( $\boldsymbol{\nabla}$ lengyueds@outlook.com )

Yunnan Cancer Hospital https://orcid.org/0000-0003-1347-5788

\section{Research Article}

Keywords: Cancer, Oncology, Cervical lesions, Psychological status, Influencing factors, Kessler 10

Posted Date: December 29th, 2021

DOI: https://doi.org/10.21203/rs.3.rs-1103852/v1

License: (c) (1) This work is licensed under a Creative Commons Attribution 4.0 International License.

Read Full License 

Investigation on psychological status of patients with cervical precancerous lesions and cancer among Han and Ethnic minority in Yunnan Province of China

Min Zhao1*, Dan Liu2*, Rong-yan Gu1, Hong-tao Lei3, Shao Zhang4, Song-rui Ding1, Lei Luo1, Meng-jiao Zhang1, Guo-yu Ma5, Zheng Li4

*Min Zhao and Dan Liu are co-first authors, and they contributed equally to this Paper.

Medical Administration Department, The Third Affiliated Hospital of Kunming Medical University (Yunnan Cancer Hospital), 519Kun Zhou Road, Xi Shan county, Kunming 650118, Yunnan, China.

2School of Public Health and Management, Chongqing Medical University, the Research Center for Medicine and Social Development, the Collaborative Innovation Center for Social Risk Governance in Health, No.1 Medical School Road, Yuzhong District, Chongqing 400016, China.

3School of Public Health, Kunming Medical University, 1168 Yu Hua Street Chun Rong Road, Cheng Gong New City, Kunming 650500, Yunnan, China. 4Department of Gynecology, The Third Affiliated Hospital of Kunming Medical University (Yunnan Cancer Hospital), 519Kun Zhou Road, Xi Shan county, Kunming 650118, Yunnan, China.

5Yunnan Cancer Center Office, The Third Affiliated Hospital of Kunming Medical University (Yunnan Cancer Hospital), 519 Kun Zhou Road, Xi Shan county, Kunming 650118, Yunnan, China.

Correspondence

Zheng Li, Department of Gynecology, The Third Affiliated Hospital of Kunming Medical University (Yunnan Cancer Hospital), 519Kun Zhou Road, Xi Shan county, Kunming 650118, Yunnan, China.

Email:lengyuedsdoutlook.com.

Funding

The National Natural Science Foundation of China (Grant Number:81760469); $i^{\circ}$ Research on Chongqing Medical Assistance System Based on i®Holistic ${ }^{-}$ Governance Perspectivei \pm project funded by Chongqing Municipal Education Commission in 2020, Grant Number: KJQN202000413; $i^{\circ}$ Research on the Overall Construction Strategy of Chongqing Medical Assistance System from the Perspective of Strategic Process Managementi \pm project funded by Chongqing Science and Technology Bureau in 2021, Grant Number: cstc2021jsyjzzysbAX0038; Postdoctoral Science Foundation of China (Grant Number:182749); The Planning Project of Philosophy and Social Sciences in Yunnan Province (Grant Number:QN2018022); Yunnan Fundamental Research Project (Grant Number:2019FE001(-085)); Innovation Team of Molecular Diagnosis and Treatment of Cervical Cancer in Kunming Medical University (Grant Number:CXTD201906); Yunnan Province i ${ }^{\circ}$ Ten Thousand People Plani \pm (Grant Number:YNWR-QNBJ-2019-099); Reserve Talents of Young and Middleaged Academic and Technical Leaders in Yunnan Province (Grant Number:2019HB049); Leader of Medical Discipline in Yunnan Province (Grant Number:D-2017052); Doctorial Innovation Fund of Kunming Medical University (Grant Number:2021D13).

Abstract: objective The aim of this study was to explore the correlation and difference of influencing factors by analyzing the psychological 
status of patients with cervical precancerous lesions and cancer in Han and Ethnic minorities. So as to provide evidence for more targeted psychological intervention for categories types of patients. Methods 200 Han patients with cervical lesions and 100 Ethnic minority patients in Yunnan cancer center were investigated with the Chinese version of Kessler 10 Scale. Statistical analysis was performed using t-test, analysis of variance, and multivariable linear regression. Results There was no significant difference in the distribution of demographic characteristics between the two groups ( $\mathrm{P}>0.05)$. The results of univariate analysis showed that the impression of K10 score was statistically significant among the following factors: educational level, awareness of HPV vaccine, disease screening status, employee medical insurance, economic burden of disease, cancerous or not, pathological type, treatment modalities, marital status, and family genetic history of tumor $(\mathrm{P}<0.05)$. Among them, the marital status had opposite effects on the two groups of patients. The mean score of married Han patients were higher than those of Han patients with other marital status, but the score of Ethnic minority patients the opposite. Multivariate analysis indicates that the economic burden of the disease, occupation, and family genetic history of tumor had a greater impact on the total score of Han patients among many factors, accounting for a total of $8.1 \% £ " A d j=0.081 £ \odot$. Treatment modalities had the greatest effect on the scores of ethnic minority patients, accounting for $8.4 \%{ }^{\prime \prime} \mathrm{Adj}=0.084 £ \odot$. Conclusion The factors affecting the psychological status of patients between the two groups have similarities and differences. Multifactorial analysis showed that the main factors affecting the psychology of Han patients were: economic burden caused by the disease, occupation, and family genetic history of tumor; while the main factors affecting the psychology of minority patients were: treatment modalities. Therefore, targeted recommendations and policy measures can be proposed respectively. We should not only increase the publicity of disease-related knowledge, but also call on all women to receive vaccines and regular screening of women in the high incidence age group. It is also necessary to positively guide patients with higher education level and give more encouragement and spiritual support to patients with family genetic history of tumor and heavy economic burden of the disease. The effect of marital relationship on patients cannot be neglected at the same time. More targeted psychological intervention and more favorable treatment modalities should be provided for patients.

Keywords: Cancer; Oncology; Cervical lesions; Psychological status; Influencing factors; Kessler 10

Introduction

Cervical cancer is a malignant tumor that occurs in the cervical canal and mainly affects people between 40 and 50 years old. The incidence and fatality rate are both increasing year by year, and are showing a younger trend [1-3]. Cervical intraepithelial neoplasia (CIN) is a precancerous lesion of invasive cervical cancer [4]. Cervical cancer is one of the most common female malignant tumors, and the global incidence ranks second among female malignant tumors [5]. According to statistics, 85\% of patients are from developing countries [6]. In 2018, cervical cancer incidence in China accounted for 18.59\% of global incidence and 15.43\% of mortality [7-8]. Due to the large population base, cultural background and lifestyle differences in China, cervical cancer has seriously threatened 
the health of women in this country. Therefore, more attention must be paid to the prevention and treatment of cervical cancer and the psychological status of patients.

More and more researchers have begun to focus on the quality of life and psychological status of patients with cervical cancer[9-13]. They explored more the current situation of psychological distress and related influencing factors [14-15], as well as the effect of social support, humanistic care, psychological intervention on the quality of life of patients [16-20]. These studies are a discussion of the integrity of the psychological status of patients, less classification and evaluation analysis of specific psychological problems, and also lack of attention to the differences in psychological status between Han and Ethnic minority patients. In this study, we used the Kessler psychological distress scale (Kessler 10) to divide the psychological status of patients into ten dimensions, and collected information from a total of 200 Han patients and 100 Ethnic minority patients from Yunnan Province as a multi-ethnic province. The economic status, lifestyle and working living environment of many ethnic minorities are quite different from those of Han nationality, which makes the psychological and mental status after suffering from cervical cancer quite different from those of Han nationality. So we focused on exploring the related factors affecting the psychological status of patients with cervical lesions and the differences in the psychological status of Han and ethnic minority patients under the influence of the same factors.

Method

Participants

Participants were 200 Han patients and 100 Ethnic minority patients with cervical lesions who were admitted and definitely diagnosed in the Third Affiliated Hospital of Kunming Medical University (Yunnan Cancer Center) from January 2019 to December 2020 were selected as respondents. Inclusion criteria: 1.aged 18 years or above, had been histologically confirmed of cervical cancer or precancerous lesions (CIN), 2.Patients with a certain educational level who can read and know the questionnaire by themselves; 3. Patients with informed consent and cooperate with the investigators; 4. Patients without mental illness and disturbance of consciousness in the past and at present. The exclusion criteria were as follows: having severe co-morbidities, mental illness, or cognitive impairment, or having other malignant tumors or serious illness at the same time, and be contraindications before radiotherapy, chemotherapy and surgery.

Material

The Kessler psychological distress scale (K10£@is a brief 10-item screen for the detection of nonspecific psychological distress widely used in international epidemiological studies of mental health dysfunction [21]. Foreign scholars have shown that $\mathrm{K} 10$ is a concise, rapid, effective and reliable mental health evaluation tool [22]. Domestic scholars have also demonstrated through research that the Chinese version of the K10 has high stability and good reliability and is suitable for the survey of the Chinese population [23].K10 includes 10 small items to evaluate people's psychological status. The content is the frequency of mental health related conditions such as psychological anxiety and psychological stress experienced in the past four weeks, which can easily detect people's 
psychological conditions and their risk factors. The Kessler 10 scale is scored from 10 to 50, with each item corresponding scores: always (5 points), usually (4 points), sometimes ( 3 points), seldom (2 points), and rarely never (1 point). The higher the score was, the worse the psychological condition was, and the greater the risk of mental health problems would be.

Procedure

This cross-sectional questionnaire survey was carried out in the years 2019.01-2020.12. All participants who consented to participate signed informed consent forms were individually interviewed, face-to-face, by trained questionnaire interviewers. First, investigate the basic demographic data of patients, including age, ethnicity (Han majority and ethnic minorities), occupation (farmer and others), level of education (primary school or below, junior high school, senior high school, college or above), marital status (unmarried, married, widowed, divorced or separated), monthly household income, Number of children, health insurance, etc, and then use the Kessler 10 scale interview respondents. The completeness of scale filling was checked after completing the survey .

Statistical

Effective questionnaire information was entered using EpiData3.1 and statistical analysis was performed with SPSS 22.0. The total score of K10 was normally distributed. Each scale score was used as a dependent variable in single factor analysis. The t-test was used to compare the sample means of two groups, and the analysis of variance was used to compare the sample means of multiple groups. We also used the multivariable linear regression analyses. The dependent variables were the scores of all items of $\mathrm{KlO}$ and the total score. Independent variables were the influencing factors in univariate analysis. Inclusion criteria

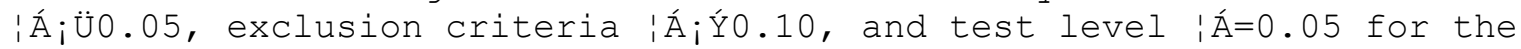
model.

Results

Basic information of participants

The distribution of Socio-demographic characteristics among Han and Ethnic minority is summarized in Table 1. A total of 300 patients with cervical lesions were investigated in this study, including 110 patients had CIN and 190 patients had Cervical cancer. The two types of patients had the same composition of the following demographic characteristics, or no statistically significant difference in distribution ( $P>0.05)$.

The Total score

As shown in Tab.2, the differences in the total scores of Han patients under the influence of disease economic status, menopause, family smoking status and family genetic history of tumor were statistically significant. Ethnic minority patients had statistically significant differences in the total score under the influence of treatment modalities, pathological types, types of health insurance, as well as cancer types. 
The score of each item

Analysis of influencing factors of K10-score

The academic community generally believes that the main factors affecting the psychological status of patients are education level, economic status, disease, and social family. Therefore, this study used methods such as t-test, analysis of variance, and multivariable linear regression to perform a single factor analysis of each score in the K10 scale. The following results are the analysis of the influencing factors with statistical significance ( $P$ i $\ddot{U}$ O.5) and summarized into four dimensions: education level and disease-related knowledge, economic status, disease types and treatment modalities, marital and family situation.

Education Level and Awareness of Disease Prevention As shown in Tab.3, the effect of education level on the boredom-score of Han patients was statistically significant, within a certain range, patients with higher educational level had higher score on this item and spent more time feeling bored. The calm-score of Ethnic minority patients was statistically significant and negatively correlated with the score, in contrast to that of Han patients. The knowledge of the vaccine had a statistically significant effect on the sitting-score and calm-score of Han and Ethnic minority patients. Whether or not to participate in the screening had a statistical significance on the calm score of all patients, as well as the difficulty-score of the Han patients, and the boredom-score of the Ethnic minority patients. The average score of the Ethnic minority patients participating in the screening were lower than those of the patients not participating in the screening.

Economic status

As shown in Tab.4, whether there was employee health insurance had a statistically significant effect on the difficulty-score and interestscore of the all patients, and also had a statistically significant effect on the-calm score of Ethnic minority patients. The impact of disease economic burden on the scores of helplessness, depression and difficulty in Han patients was statistically significant, and the higher the patienti ${ }^{-}$s score, the heavier the economic burden.

Disease types and Treatment modalities

As shown in Tab.5, Whether cancerous had a statistically significant effect on the difficulty-score of all patients, and also had a statistically significant effect on the sitting-score, rest-score, helplessness-score, interest-score of Ethnic minority patients, and the average score of cervical cancer patients were higher Han those of CIN patients. The effect of pathological type on the calm-score, rest-score, helplessness-score, difficulty-score, interest-score of Ethnic minority patients was statistically significant. The mean score of squamous cell carcinoma patients were higher than patients with other pathological types, but there was no significant effect on the score of Han patients. The impact of treatment modalities on the calm-score, rest-score, helplessness-score, difficulty-score of Ethnic minority patients was statistically significant. The mean score of patients treated with Concurrent chemoradiation and postoperative adjuvant therapy were higher than those of patients treated with other methods, but the effect on the score of Han patients was also not statistically significant. 
Marital and family situation

As shown in Tab.6, the effect of marital status on the sitting score and calm score of Han patients was statistically significant. The mean score of married Han patients was higher than those of Han patients with other marital status. The effect on the exertion-score and interest-score of Ethnic minority patients was statistically significant, and the mean score of Ethnic minority patients with other marital status was higher than those of married Ethnic minority patients. Family genetic history of tumor had a statistically significant effect on the tension-score and interest-score of Han patients, and a statistically significant effect on the helplessness-score of Ethnic minority patients. The mean score of patients with family genetic history of tumor were higher than those of patients without family genetic history of tumor.

The total score results of multivariable linear regression analysis of influencing factors

As shown in supplementary Table 1, the total score was associated with economic burden of disease $(\hat{A}=0.288, P<0.001)$, occupation $(\mid \hat{A}=$ $0.192, \mathrm{P}=0.012)$, and family genetic history of tumor $(\hat{A}=$ ? $1.44, \mathrm{P}=$ $0.036)$ in Han patients. The total score in minority patients was associated with treatment modalities $(i \hat{A}=0.305, P=0.002)$.

\section{Discussion}

Education Level and Awareness of Disease Prevention There were significant differences in the effects of education level and Disease screening status of Han patients and ethnic minority patients. Higher education level and certain knowledge of disease prevention help alleviate the psychological problems of Ethnic minority patients, especially can help patients recover calm in stressful emotions. And all psychological status indicators of ethnic minority patients who participated in the screening were better than those of patients who did not participate in the screening. This is the same with the research conclusion of Chinese scholar Wu Ying [24]. Some studies [25-26] have also found that the quality of life of cervical cancer survivors is related to their education level. The higher the education level, the higher the quality of life of patients. This is related to the occurrence and development of cervical cancer. Cervical lesions are a continuous process, which takes several years to more ten years from precancerous lesions to invasive cancer. Some early lesions can be reversed [27]. Timely vaccination and regular participation in screening are helpful for the treatment and outcome of the disease. However, the education level is low, the ability to accept knowledge is limited, and the knowledge of diagnosis and treatment related to cervical cancer is less. So it is difficult to make a correct and objective understanding of the disease and treatment, and the awareness of disease prevention is also insufficient. However, the results showed that illiterate patients had the lowest scores in both Han and ethnic minority patients. And the psychological status of Han patients did not become better completely with increasing education level. Among them, the scores of patients with high school degree showed a significant increase£" $\mathrm{M}=1.750 £ \neg \mathrm{SD}=0.794 £ \odot$, and the scores of college and above decreased to the lowest scores of patients except illiterate $(\mathrm{M}=1.350 £ \neg \mathrm{SD}=0.587 £ \odot$. This may be due to the fact that illiterate patients do not pay enough attention to the disease, 
lack awareness of prevention, and the disease does not burden their psychology. Patients with moderate education levels, on the other hand, are more aware of the disease, but it is not comprehensive and scientific enough. Eventually, some patients produce negative emotions, lose enthusiasm for things and life, and aggravate their psychological problems. With the further improvement of cultural level, the understanding of disease and health is more perfect and scientific, and mental health has also been improved.

Therefore, we should strengthen the publicity of cervical cancer-related knowledge and call for regular screening of women in the vaccinated and high incidence age groups. At the same time, it is also necessary to provide simpler and more detailed guidance for patients with low education level, especially real Ethnic minority patients. It is also necessary to explain the clinical symptoms of the disease and the adverse reactions of postoperative chemotherapy to patients with a moderate education level of education, and provide positive guidance to enhance patients' confidence in treatment, thereby improving their psychological state $[28-29]$.

Economic status

Family economic status is an important factor affecting the quality of life of patients [30]. The Han patients with heavier disease economic burden had higher scores and more time to feel helpless, depressed and difficult to do things. This result is the same as the results of multiple studies [31-32]. This is because the treatment of cervical cancer is a long-term process, with complex treatment modalities and high treatment cost. Besides, chemotherapeutic drugs also have toxic and side effects, which can impair the body function and must be relieved by drugs, leading to an increase in the economic burden. In a poor economic situation, patients may take less drugs, resulting in the aggravation of toxic and side effects [33], which has caused severe physical and psychological suffering. At the same time, the disease may also affect the patients' daily work and life, or even lose their jobs, further aggravating the economic burden of the disease. Medical insurance can also reflect the economic burden of disease. From the impact of employee medical insurance on Ethnic minority patients, it is found that the total score of K10 of the patients with employees' insurance reimbursement is 14.8, which is lower than the patients with non-employee medical insurance 16.6. Li Weiling's study [32] also found that patients whose costs were mainly derived from health insurance had better mental health status than those from other patients. Although the effect of having employee medical insurance on Han patients is not uniform, the psychological effect of economic burden on all patients cannot be ignored. Therefore, we also need the support of the national medical insurance system and the joint efforts of all sectors of society to try our best to reduce treatment costs, increase the coverage and proportion of medical insurance reimbursement, and reduce the economic pressure of patients' diseases to alleviate their psychological problems.

Disease types and Treatment modalities It is found that the total score of Klo of the Ethnic minority patients with carcinogenesis, especially squamous cell carcinoma is 18.7, which is higher than the patients with Adenocarcinoma 12.5. And patients with cancer find it difficult to do anything more time. This is because, not 
only cervical cancer patients will produce symptoms of severe anxiety and depression during chemoradiotherapy, but also patients' psychological adverse emotions cannot be avoided during neoadjuvant chemotherapy for cervical cancer. Chemoradiotherapy brings pain to the body and also affects their psychological status. During chemoradiotherapy, adverse reactions such as gastrointestinal reactions and alopecia will make patients suffer from physical and psychological torture, resulting in reduced treatment enthusiasm and compliance. In addition, high chemotherapy cost will aggravate its psychological pressure [34].The patients with Cervical conization and radical hysterectomy had lower scores. Zheng Yingying's study [35]pointed out that surgery is the best early treatment for patients with cervical cancer, which can effectively improve the life cycle of patients, but at the same time will have an impact on patients, such as physiological defects caused by hysterectomy. So formulating the treatment plan for patients, these two methods could be given priority in a scientific and reasonable case to reduce the psychological problems of patients after treatment. The pathological type and treatment modalities had a greater impact on Ethnic minorities, so more attention and psychological counseling could be given to Ethnic minority patients with squamous cell carcinoma.

Marital and family situation

In China, the family is a very important social system and a strong spiritual pillar, and the role of women in the family is very special. Compared with married patients, patients with other marital conditions lack the care and support from the other half or children, as well as the sense of belonging and identity from the family, will bear more loneliness and helplessness. Some scholars have shown [36] that sexual life has a certain impact on the quality of life of patients with gynecological malignancies. And the earlier sexual life is started after surgery, the faster the negative impact caused by surgery and chemoradiotherapy can be relieved, helping patients eliminate their doubts about their female identity. However, some scholars also point out that compared with other types of tumors, gynecological tumors have the greatest negative impact on female sexual behavior and will last for a period of time [37]. Approximately 70\% of cervical cancer survivors suffer from sexual dysfunction, such as decreased vaginal sensitivity, reduced sexual desire and sexual excitement in patients due to vaginal dryness, pain bleeding, dyspareunia, and vaginal atrophy after treatment [38].

Even some patients had to remove the uterus during the treatment, which leads to physiological problems such as sexual dysfunction and loss of reproductive function [39], other treatment modalities such as concurrent chemoradiotherapy will also cause physiological damage to a certain extent. The treatment of cervical cancer requires a long recovery period, which will lead to decreased sexual desire and even sexual dysfunction of patients, affecting the life of husband and wife and destroying family relations. It instead causes the patient to fall into a dilemma of anxiety and helplessness. The analysis results showed that there were large differences in the scores of Han and Ethnic minority patients in different marital states. Married Ethnic minority patients had less time to feel exhausted and considered life boring than Han patients with other marital status. Married Han patients scored higher, more time feel restless, difficult to calm. Therefore, we should pay attention not only 
to the patient's psychological status but also to the patient's other half of the mood as well as the husband-wife relationship. Medical staff should help patients correctly face sex-related problems and provide necessary sexual life guidance to help them improve their sexual life status [40].Having a loving sexual life and a harmonious family relationship is conducive to the rehabilitation of patients.

CONCLUSIONS

Our study identified the factors affecting the psychological status of patients between the Han and Ethnic minorities were different. Multifactorial analysis showed that the main factors affecting the psychology of Han patients were: economic burden caused by the disease, occupation, and family genetic history of tumor; while the main factors affecting the psychology of Ethnic minority patients were: treatment modalities. Therefore, targeted recommendations and policy intervention measures can be proposed respectively. We should increase awareness about cervical cancer and call for women to be vaccinated, in particular, for women in the high-incidence age group to receive regular screening. Easier and more detailed instructions need to be provided for patients with low education levels, especially for Ethnic minority patients. It is necessary to give more spiritual support to patients with family genetic history of tumor and heavy economic burden of the disease. Treatment modalities have a greater impact on Ethnic minorities, and more attention and psychological counseling should be given to them.

1. Study limitations

This study has some limitations. Firstly, the sample size is small, and a single center study. Secondly, the cross-sectional study design employed means causal conclusions could be made with caution. Thirdly, other factors such as the prevalence of the living environment, behavior habits and the quality of life were not considered. Finally, blank control is absent in this study.

2. Clinical implications

The current study suggested that it should not only increase the publicity of disease-related knowledge, but also call on all women to receive vaccines and regular screening of women in the high incidence age group. It is also necessary to positively guide patients with higher education level and give more encouragement and spiritual support to patients with family genetic history of tumor and heavy economic burden of the disease. More targeted psychological intervention and more favorable treatment modalities should be provided for patients.

Conflicts of interest

The authors declare that there are no conflicts of interest.

Availability of data and material

The data used for the current study are available from the corresponding author on reasonable request.

Code availability

Not applicable.

Authors' contributions

Min Zhao*: Designed the study, and guided writing.

Dan Liu*: Conceptualization, and wrote the main manuscript. 


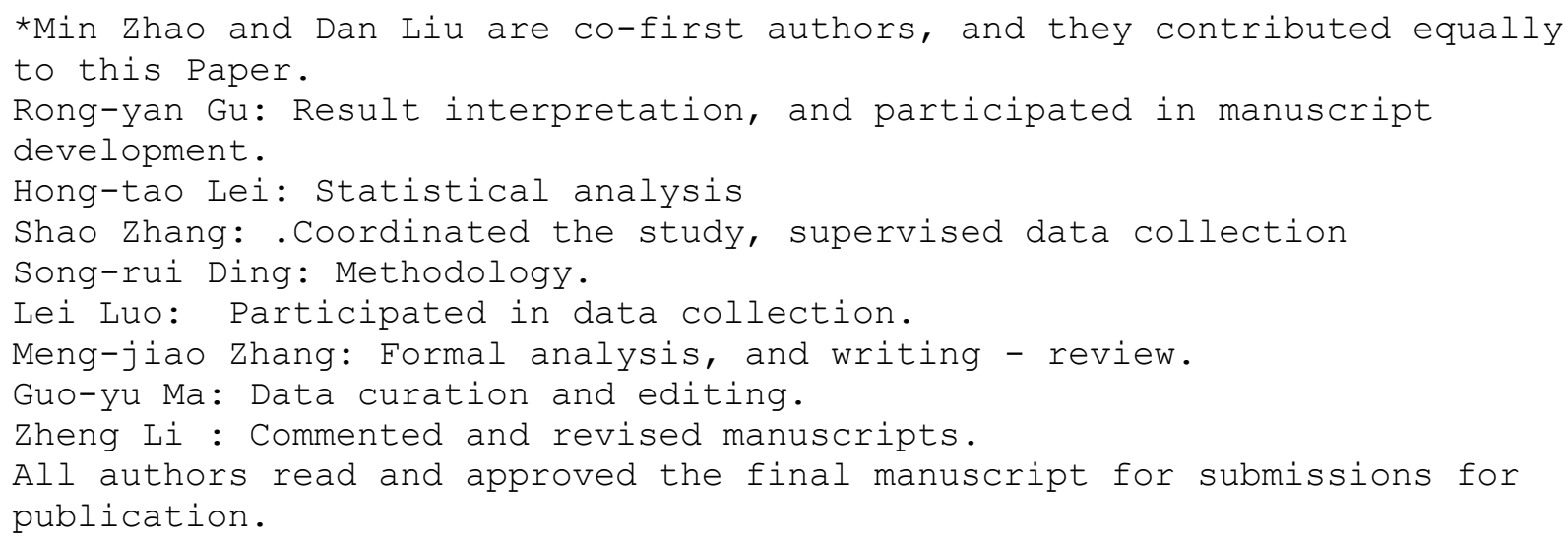

REFERENCES 
1. Zhou L, Meng Q, Yang Z, Li G, Yang H Y, Zhang H P. Analysis of factors influencing quality of life of cervical cancer patients based on FACT-Cx(V4.0) scale[J]. Chinese Journal of Disease Control, 2017,21(09):926-929+938.

2. Li $\mathrm{S}$, et al. Changes in prevalence and clinical characteristics of cervical cancer in the People's Republic of China: a study of 10,012 cases from a nationwide working group. Oncologist, 2013. 18(10): 11011107 .

3. Wanqing Chen PhD, MD, Rongshou Zheng MPH, Peter D. Baade PhD, Siwei Zhang BMedSc,Hongmei Zeng PhD, MD, Freddie Bray PhD, Ahmedin Jemal DVM, PhD, Xue Qin Yu PhD, MPH, Jie He MD. Cancer statistics in China, 2015[J].

4. Peng Qiyan. Study on the clinical value of HPV genotyping screening for cervical lesions in 11455 cases in Yingcheng City[J]. China Experimental Diagnostics, 2020,24(10):1665-1667.

5. Xiao L, Yang Xuejun, Wang Hongjing, Xiao Songyuan, Wang Lei, Ren Yanjie, Yuan J, Liu Shaoli. A survey on cervical cancer awareness, attitude and behavior among 608 community women in Chengdu[J]. Modern preventive medicine, 2013,40(4):706-709.

6. Vaccarella $\mathrm{S}$, et al. Cervical cancer in Africa, Latin America and the Caribbean and Asia: Regional inequalities and changing trends. Int. J. Cancer, 2017. 141(10): 1997-2001. 7. BRAY F, FERLAY J, SOERJOMATARAM I, et al. Global cancerstatistics 2018: GLOBOCAN estimates of incidence and mortalityworldwide for 36 cancers in 185 countries£ÛJ£Ý. CA Cancer J Clin, 2018,68(6):394-424. 8. ARBYN M, WEIDERPASS E,BRUNI $\mathrm{L}$, et al. Estimates of inci-dence and mortality of cervical cancer in 2018: a worldwide anal-ysis£ÛJ£Ý.Lancet Glob Health, 2020,8(2):e191-e203.

9. Leath Charles A, Monk Bradley J. Twenty-first century cervical cancer management: A historical perspective of the gynecologic oncology group/NRG oncology over the past twenty years.[J]. Gynecologic oncology, 2018,150(3).

10. Tsai LY, Lee SC, Wang KL, et al.A correlation study of fear of cancer recurrence, illness representation, self-regulation, and quality of life among gynecologic cancer survivors in Taiwan[J]. Taiwan J Obstet Gynecol, 2018,57(6):846-852.

11. Zhou H, Liu J. Study on economic burden, anxiety and depression and corresponding psychological care of gynecological malignancy cancer patients[J]. Hebei Medicine, 2020, 42(6):158-161.

12. Patel H, Moss EL, Sherman SM.HPV primary cervical screening in England: Women's awareness and attitudes[J].Psychooncology,2018,27(6):1559-1564. 13. Yi-Long Yang, Li Liu,Xiao-Xi Wang, Yang Wang, Lie Wang. Prevalence and Associated Positive Psychological Variables of Depression and Anxiety among Chinese Cervical Cancer Patients: A Cross-Sectional Study[J]. PLOS ONE, 2014, $9(4)$.

14. Li Yuanfang, Zhang Jun'e. Investigation and analysis of psychological distress of cervical cancer patients and its influencing factors [J]. Integrated Chinese and Western Medicine Nursing(Chinese and English), 2020,6(8):172-178.

15. Li W L, Chen Y Y, Tang X F, Tan H, Tao Y, Liu Y Y. Survey on the current situation of psychological distress of cervical cancer patients and analysis of related factors[J]. Journal of Advanced Nurse Training, 2019,34(24):2209-2213. 
16. Cheng R. H. Exploring the impact of psycho-behavioral interventions on psychological health and quality of life of cervical cancer patients $[\mathrm{J}]$. Chinese Journal of Health Psychology,2018,26(8):1152-1156. 17. Hu Ying. Study on the role of psychological care in the treatment of cervical cancer [J]. China Medical Guide,2020,18(29):197-198.

18. An S F, Li S Y, LV J, Ren H, Wang X L. Correlation analysis of social support and quality of life of cervical cancer patients[J]. China Maternal and Child Health Research,2017,28(3):351-353. 19. Zhang W W W, Ji Y Y, Xiang M, Li X L. Effects of humanistic therapeutic communication on postoperative recovery, negative emotions and marital quality in patients with cervical cancer[J]. Chinese Journal of Health Psychology:1-11.

20. Wu L P, Zhou J J, Li N. Exploration on the application of humanistic care in the care of cervical cancer chemotherapy patients [J]. Heilongjiang Traditional Chinese Medicine, 2020,49(6):346-347. 21. Kessler RC, Green JG, Gruber MJ, et al. Screening for serious mental illness in the general popula-tion with the K6 screening scale: results from theWHO World Mental Health (WMH) survey initiative[J]. Int J Methods Psychiatr Res, 2011,20(1):62.

22. Lace JW, Greif TR, Mcgrath A, et al. Investigatingthe factor structure of the K10 and identifying cut-off scores denoting nonspecific psychological dis-tress and need for treatment[J]. Mental Health \&Prevention, 2019,13:100-106.

23. Zhou Chengchao, Chu Jie, Wang Ting, Peng Qianqian, He Jiangjiang, Zheng Wengui, Liu Dongmei, Wang Xingzhou, Ma Hongfeng, Xu Lingzhong. Reliability and validity evaluation of the Chinese version of the Kessler 10 Brief Psychological Profile Rating Scale[J]. Chinese Journal of Clinical Psychology, 2008,16(6):627-629.

24. Wu Y, Kong J, Wang S L, Zhang M. Psychological impact of positive cervical cancer screening results on women[J]. Contemporary Medicine, 2021,27 (9):59-61. 25. Vincer?evskiene Ieva, Jasilionis Domantas, Austys Donatas, Stukas Rimantas, Kaceniene Auguste, Smailyte Giedre. Education predicts cervical cancer survival: a Lithuanian cohort study.[J]. European journal of public health, 2017,27(3).

26. SMAILYTEG, JASILIONISD, VINCERZEVSKIENEI, et al. E-ducation, survival, and avoidable deaths in Lithuanian cancer pa-tients, 2001$2009 £ \hat{U} J £ Y ́$. Acta Oncol, 2016, 55 (7):859-864.

27. Xie Aijun. Analysis of screening results of 11598 rural cervical cancer cases in Lin'an City [J]. China Maternal and Child Health Care, 2015,30(2):684-685.

28. Qi Jingjing, Hou Qian, Luo De Feng, Qiu Shuangfeng. Effects of pharmacological interventions on chemotherapy-induced nausea, vomiting and negative emotions and quality of life in breast cancer patients[J]. Guizhou Medicine,2019,43(10):1550-1552.

29. Sun Yeqi, Wang Ruifen, Qiao Meng, Xu Yanchun, Guan Wenbin, Wang Lifeng. Cancer associated fibroblasts tailored tumor microenvironment of therapy resistance in gastrointestinal cancers.[J]. Journal of cellular physiology, 2018,233(9). 30. Reis Nathalia V S, Andrade Brenda B, Guerra Maximiliano R, Teixeira Maria Tereza B, Malta Deborah C,Passos Val“'ria M A. The Global Burden of Disease Study Estimates of Brazil's Cervical Cancer Burden. [J]. Annals of global health, 2020,86(1). 
31. Lao C. M.,Wu H. Y. Investigation of psychological status of patients with postoperative chemotherapy for cervical cancer and analysis of related factors[J]. Zhejiang Journal of Integrative Medicine, 2020,30(9):771-774. 32. Li W L, Chen Y Y, Tang X F, Tan H, Tao Y, Liu Y Y. Survey on the current situation of psychological distress of cervical cancer patients and analysis of related factors[J]. Journal of Nursing

Advancement, 2019,34(24):2209-2213.

33. Qian Rong, Lian Xiangqun, Lin Yuxia. A longitudinal study of quality of life and its influencing factors during chemotherapy in patients with cervical cancer[J]. Nursing Practice and Research,2020,17(3):8-11. 34. Zhou $Q$. Impact of nursing interventions on survival quality in patients undergoing chemotherapy for cervical cancer [J]. Journal of Liberation Army Hospital Management, 2017,24(3):285-287+291. 35. Zheng YY, Lu HY, Chen ZF, et al. A qualitative study of patients' symptom experience and psychological feelings during the recovery period after radical cervical cancer surgery[J]. Chinese Journal of Nursing, $2020,55(4): 569-573$.

36. Du Ang-Ying, Zhang Jia-Wen, Tang Lei, Yu Mei-Jia. Investigation of postoperative survival quality in patients with gynecologic malignancies[J]. Chinese Journal of Practical Gynecology and Obstetrics, 2007, (3):198-200.

37. Rajeev Ramchand, Sangeeta C. Ahluwalia, Lea Xenakis, Eric Apaydin, Laura Raaen, Geoffrey Grimm. A systematic review of peer-supported interventions for health promotion and disease prevention[J]. Preventive Medicine, 2017,101.

38. Zehra Golbasi, Ayten Senturk Erenel. The quality of sexual life in women with gynaecological cancers[J]. Archives of Gynecology and Obstetrics, 2012,285 (6).

39. Jiang Qiangying, Ma Xiaoxia. Analysis of survival quality of patients with gynecologic malignancies after surgery and its influencing factors[J]. Clinical Medicine Research and Practice, 2019,4(11):83-84. 40. Xia Xuan, Li Dan, Cheng Lin, Duan Juan, Shi Yan. Research progress on the quality of survival of patients with cervical cancer[J]. Shanghai Nursing, 2021,21(2):45-49. 


\section{Supplementary Files}

This is a list of supplementary files associated with this preprint. Click to download.

- SUPPLE1.jpg 